\title{
Terahertz Generation from Femtosecond-Laser-Excited GaAs Surface Due to Electric-Field-Induced Optical Rectification
}

\author{
V.L. MALEViCH ${ }^{a}$, P.A. Ziaziulia ${ }^{b, *}$ AND I.S. MANAK $^{b}$ \\ ${ }^{a}$ B.I. Stepanov Institute of Physics \\ National Academy of Sciences of Belarus \\ Nezalezhnasti Av. 68, 220072, Minsk, Belarus \\ ${ }^{b}$ Belarussian State University, Nezalezhnasti Av. 4, 220050, Minsk, Belarus \\ The dynamics of the depletion field screening induced by photoexcited \\ carriers and $\mathrm{THz}$ generation caused by the electric-field-induced optical rec- \\ tification are simulated for GaAs surface excited by femtosecond laser radia- \\ tion on the basis of an ensemble Monte Carlo method. The results show that \\ the photocarrier-induced screening occurs on a subpicosecond time scale and \\ $\mathrm{THz}$ pulse essentially changes its wave form depending on excitation pulse \\ duration and fluence. The possibility to use the depletion electric field in- \\ duced $\mathrm{THz}$ generation for study of subpicosecond electric field screening \\ dynamics is discussed.
}

PACS numbers: 42.65.Re, 42.65.Ky, 73.30.+y

\section{Introduction}

Semiconductor surfaces irradiated with femtosecond laser pulses can emit electromagnetic radiation in terahertz $(\mathrm{THz})$ frequency region. It has been established that $\mathrm{THz}$ radiation is associated with a dipole that is induced near the photoexcited semiconductor surface due to a transient photocurrent surge and (or) nonlinear polarization following the femtosecond laser pulse envelope. Traditionally, it is supposed that $\mathrm{THz}$ emission from GaAs results from a transient photocurrent driven by the built-in electric field due to a band bending near semiconductor surface. However, at high excitation fluence the photocurrent saturates and the optical rectification can contribute considerably to the $\mathrm{THz}$ generation.

${ }^{*}$ corresponding author; e-mail: palz@tut.by 
In the non-centrosymmetric semiconductors like GaAs the nonlinear optical polarization responsible for the optical rectification effect consists of two parts, intrinsic bulk and electric-field-induced $[1,2]$, and is given by

$$
P_{i}=\chi_{i j k}(0 ; \omega,-\omega) E_{j} E_{k}^{*}+\chi_{i j k l}(0 ; \omega,-\omega, 0) E_{j} E_{k}^{*} F_{l},
$$

where $\chi_{i j k}$ and $\chi_{i j k l}$ are the second- and third-order nonlinear susceptibilities, $\omega$ and $E$ are the frequency and the amplitude of optical field. The third-order term in (1) containing the built-in surface electric field $F$ describes the so-called electric-field-induced optical rectification (EFIOR) effect.

The distinguishing feature of $\mathrm{THz}$ emission resulting from the optical rectification is its dependence on the orientation of pump radiation electric field with respect to the crystallographic axis (azimuthal anisotropy). It has been pointed out that the bulk and EFIOR contributions can be distinguished due to their different angle dependences; for example, in zinc-blende semiconductors for (001) crystalline surface the THz signal is independent of azimuthal angle $\varphi$ for EFIOR mechanism and changes, as $\cos 2 \varphi$ for bulk mechanism.

Photocarrier transport in depletion layer is accompanied by the charge redistribution and electric field screening. The time scale of these processes depends on semiconductor parameters (doping level, momentum relaxation rate) and excitation fluence, and is usually of the order of $10^{-13} \mathrm{~s}$. Thus, under femtosecond laser excitation the built-in surface electric field leading to EFIOR effect can change essentially over the excitation pulse duration and hence the electric field screening effect should be taken into account. In this paper we present the results of Monte Carlo simulation of depletion electric field dynamics at GaAs surface excited with femtosecond laser pulse and $\mathrm{THz}$ emission stemming from EFIOR effect.

In the far field the THz field can be expressed as

$$
E_{\mathrm{THz}} \approx \frac{8 \pi S \chi^{(3)}}{c^{3} n R} \frac{\mathrm{d}^{2}}{\mathrm{~d} t^{2}}\left(I(t) \int_{0}^{\infty} F(z, t) \mathrm{e}^{-\alpha z} \mathrm{~d} z\right),
$$

where $I(t)=c n E^{2} / 8 \pi$ is the laser pulse intensity, $c$ is the light velocity in vacuum, $n$ and $\alpha$ are the refractive index and the absorption coefficient of the pump radiation, respectively, $R$ is the distance from the illuminated spot with the area $S$ to the observation point; the effective nonlinear susceptibility $\chi^{(3)}$ depends on the angle of incidence, linear refractive index, crystal orientation, and the third-order susceptibility components $\chi_{i i i i}$ and $\chi_{i j i j}\left(=\chi_{i j j i}=\chi_{i i j j}\right)$.

To describe carrier transport and dynamics of the depletion field on a subpicosecond time scale we use a macro-particle method [3]. This method is based on an ensemble Monte Carlo simulation of carrier transport in time-varying inhomogeneous electric field that is found self-consistently from one-dimensional Poisson equation.

Specific calculations were carried out for $1.6 \times 10^{15} \mathrm{~cm}^{-3} n$-type GaAs irradiated by femtosecond laser pulse with the photon energy $1.55 \mathrm{eV}$ and $\mathrm{sech}^{2}$ temporal wave form. 


\section{Results and discussion}

The calculated evolution of the depletion layer electric field is presented in Fig. 1 for two excitation fluencies. One can see that with increase in the excitation level the character of the depletion field temporal decay changes from monotonic to oscillatory, which can be explained as changing the transport regime from hydrodynamic to collisionless one [4]. The frequency of the oscillations is determined by the plasma frequency which depends on the total density of electrons (including equilibrium and photoexcited one). The oscillatory behavior of the field screening is realized in the case that the plasma frequency exceeds the electron momentum relaxation rate.
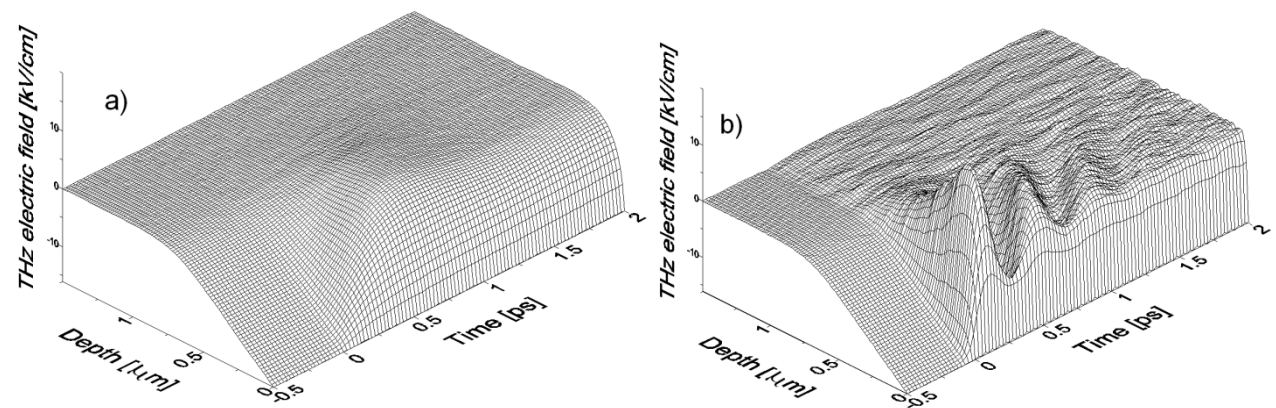

Fig. 1. Simulated evolution of the depletion field at GaAs surface excited by $100 \mathrm{fs}$ laser pulse with fluences of $0.1 \mu \mathrm{J} / \mathrm{cm}^{2}$ (a) and $1 \mu \mathrm{J} / \mathrm{cm}^{2}$ (b).

The temporal wave forms of $\mathrm{THz}$ field calculated for the different pump pulse durations are shown in Fig. 2a. With decreasing the pulse duration the influence of the photocarrier-induced screening on the THz-pulse shape becomes of minor importance since the depletion electric field does not manage to vary substantially over the pulse duration. Fig. $2 \mathrm{~b}$ shows the temporal dependences of $\mathrm{THz}$ wave forms calculated for the different excitation fluencies. It is seen that the $\mathrm{THz}$ electric field quadratically depends on the fluence and changes its polarity at high excitation level.

$\mathrm{THz}$ emission induced by the EFIOR effect can be useful for investigation of subpicosecond dynamics of the built-in field screening and can have some advantages over the electric-field-induced second harmonic ( $\mathrm{SH})$ generation [5]. The point is that SH signal is generated only in the near-surface layer with the depth of about $10 \mathrm{~nm}$ (the absorption length of $\mathrm{SH}$ ). In this region the built-in surface electric field slightly changes because it is determined by the density of carriers occupying the surface states which has not changed during femtosecond excitation. On the other hand, THz-pulse is emitted by nearly whole depletion layer because its typical width is comparable to the penetration depth of the pump radiation. To study the ultrafast dynamics of the built-in electric field in photoexcited semiconductors the pump-probe technique can be used [6]. In this method 

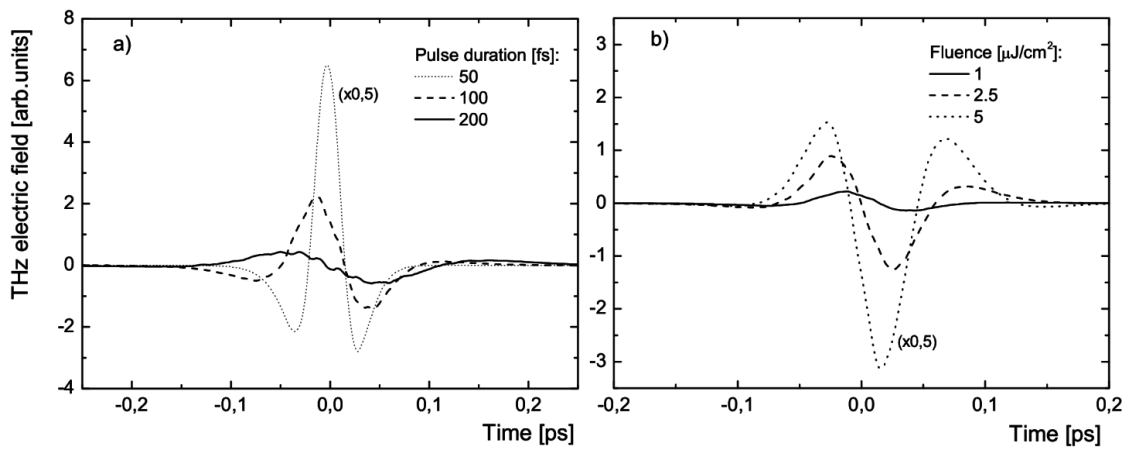

Fig. 2. THz electric field calculated for different pulse durations (a) and different fluencies (b).

the photocarrier-induced surface field screening is initiated by the pump pulse. The wave form of $\mathrm{THz}$ field generated by the probe pulse following the time delay relative to the pump pulse depends on the depletion field.

\section{Conclusions}

In conclusion, we have analyzed $\mathrm{THz}$ pulse generation that was caused by the optical rectification induced by the depletion field at GaAs surface. The simulation carried out with the macro-particles method shows that due to the ultrafast spatial redistribution of photoexcited carriers the surface depletion field can vary substantially during the course of the exciting pulse and, as a result, the photocarrier-induced field screening can essentially influence the THz-pulse wave form. Thus, the EFIOR effect in $\mathrm{THz}$ pulse generation can be used to study the dynamics of the built-in electric field screening in photoexcited semiconductors on a subpicosecond time scale.

\section{References}

[1] R. Adomavicius, A. Urbanowicz, G. Molis, A. Krotkus, E. Satkovskis, Appl. Phys. Lett. 85, 2463 (2004).

[2] M. Reid, I.V. Cravetchi, R. Fedosejevs, Phys. Rev. B 72, 035201 (2005).

[3] R.W. Hockney, J.W. Eastwood, Computer Simulation Using Particles, McGrawHill, New York 1981.

[4] V.L. Malevich, Semicond. Sci. Technol. 17, 551 (2002).

[5] D. Lim, M.C. Downer, J.G. Ekerdt, Appl. Phys. Lett. 77, 181 (2000).

[6] A. Krotkus, R. Adomavicius, G. Molis, V.L. Malevich, J. Nanoelectron. Optoelectron. 2, 108 (2007). 